\title{
Estructura de las juntas directivas y divulgación de la responsabilidad social corporativa: El caso de las empresas más reputadas en Colombia*
}

\author{
Structure of the boards of directors and disclosure of corporate social responsibility: The case of the most \\ reputable companies in Colombia \\ Estrutura dos conselhos de administração e divulgação da responsabilidade social corporativa: $\mathrm{O}$ caso das \\ empresas mais conceituadas da Colômbia
}

Alejandro Wilches Segovia

Universidad Externado de Colombia, Colombia

ORCID: https://orcid.org/0000-0003-3482-9186

Luis Antonio Orozco Castro ${ }^{\text {a }}$

Universidad Externado de Colombia, Colombia

luis.orozco@uexternado.edu.co

ORCID: https://orcid.org/0000-0001-7526-4500

Cesar Yamit Beltrán Torres

Universidad Externado de Colombia, Colombia
DOI: https://doi.org/10.11144/Javeriana.cao33.ejdd

Recibido: 01 Octubre 2018

Aceptado: 19 Agosto 2020

Publicado: 20 Diciembre 2020

\section{Resumen:}

Las grandes empresas buscan mejorar su legitimidad desde la revelación de información sobre su gestión no financiera en responsabilidad social corporativa (RSC). Sin embargo, la relación entre la estructura de la junta directiva y una mayor revelación de indicadores ambientales y sociales, dada una estructura de propiedad y un nivel de reputación corporativa, no ha sido estudiada, especialmente en economías emergentes. Usando el debate entre la teoría de la agencia y de dependencia de recursos con una muestra de 115 empresas listadas en Merco Colombia, esta investigación concluye con un análisis multivariado que el tamaño, la independencia y los comités de ética, gobierno y sostenibilidad inciden en la revelación en el Global Reporting Initiative (GRI) sobre RSC en empresas no familiares con alta reputación.

Códigos JEL: G32, G34, L25, M14, Q56.

Palabras clave: Gobierno Corporativo, Junta Directiva, Responsabilidad Social Corporativa, Sostenibilidad, Reputación Corporativa, GRI, Empresa Familiar, Reporte no Financiero.

\section{Abstract:}

Large companies seek to improve their legitimacy through the disclosure of information about their non-financial management in corporate social responsibility (CSR). However, the relationship between the board structure and a greater disclosure of environmental and social indicators, given an ownership structure and a level of corporate reputation, has not been studied, especially in emerging economies. Using a debate between agency and resource dependence theory with a sample of 115 companies listed in Merco Colombia, this research concludes with a multivariate analysis that size, independence, and ethics, governance and sustainability committees affect the disclosure in the Global Reporting Initiative (GRI) on CSR in non-family businesses with a strong reputation.

JEL Codes: G32, G34, L25, M14, Q56.

Keywords: Non-financial Reporting, Corporate Governance, Board of Directors, Corporate Social Responsibility, Sustainability, Corporate Reputation, GRI, Family Business, Non-financial Reporting.

\section{Resumo:}

As grandes empresas buscam melhorar sua legitimidade a partir da divulgação de informações sobre sua gestão não financeira em responsabilidade social corporativa (RSC). No entanto, a relação entre a estrutura do conselho de administração e uma maior divulgação de indicadores ambientais e sociais, dada uma estrutura de propriedade e um nível de reputação corporativa, não foi estudada, especialmente em economias emergentes. Usando o debate entre a teoria da agência e da dependência de recursos com uma amostra de 115 empresas listadas no Merco Colômbia, esta pesquisa conclui com uma análise multivariada que o tamanho,

Notas de autor

a Autor de correspondencia. Correo electrónico: luis.orozco@uexternado.edu.co 
independência e comitês de ética, governança e sustentabilidade afetam a divulgação no Global Reporting Initiative (GRI) sobre RSC em empresas não familiares com grande reputação.

Códigos JEL: G32, G34, L25, M14, Q56.

Palavras-chave: Relatório Não Financeiro, Governança Corporativa, Conselho de Administração, Responsabilidade Social Corporativa, Sustentabilidade, Reputação Corporativa, GRI, Empresa Familiar, Relatório Não Financeiro.

\section{Introducción}

Las empresas tienden a revelar no solo su situación financiera, sino sus resultados en la gestión enmarcada dentro de la responsabilidad social corporativa -RSC-. Los indicadores sociales y medioambientales que dan cuenta de la capacidad empresarial por aportar a la sostenibilidad contribuyen a mejorar la legitimidad basada en la percepción positiva de diversos grupos de interés (Patten, 2002, 2019). En este contexto, la rendición de cuentas sobre los resultados financieros y no financieros es uno de los requerimientos que vienen posicionándose en el plano institucional (Orozco, Vargas, \& Galindo, 2018).

Uno de los medios más importantes para la legitimación está en la revelación de información a partir de estándares como el del Global Reporting Initiative -GRI-, que orienta a las organizaciones con un énfasis metodológico práctico para dar cuenta de su desempeño ambiental, social y económico. También se cuenta con el marco del Consejo Internacional de Reporte Integrado -IIRC, por sus siglas en inglés-, que motiva a las organizaciones a llevar a cabo un proceso de reporte que refleje la estrategia de negocio y la creación de valor desde un pensamiento integrado y la gestión activa de los capitales financiero, industrial, intelectual, humano, social y relacional y natural.

Estas guías y lineamientos se han convertido en un requisito de revelación de información social, ambiental y económica cada vez más exigente, que se articulan con la orientación de las empresas hacia la organización de un sistema de gobierno corporativo sólido. La relación entre el gobierno corporativo-GC-con particular referencia al rol de la junta directiva y la divulgación sobre RSC ha sido abordada para el sector bancario (Jizi et al., 2014), empresas del Fortune 500 (Giannarakis, 2014) e incluso comparaciones entre varios países (Fernandez-Feijoo, Romero, \& Ruiz-Blanco, 2014; Michelon \& Parbonetti, 2012). Sin embargo, la revelación de la información social y ambiental comparativamente con las características de la junta directiva, la reputación corporativa y la estructura de propiedad en términos de empresas familiares es un tema emergente en países de América Latina.

En Colombia se han llevado a cabo algunas iniciativas de rendición de cuentas y buenas prácticas empresariales, lo que ha incidido en el mejoramiento de la composición de las juntas directivas y el desempeño y cumplimiento (Herrera-Echeverri et al., 2018). Por ejemplo, la Encuesta Código País de la Superintendencia Financiera y la Encuesta de Gobierno Corporativo y Responsabilidad Social Corporativa de la Superintendencia de Sociedades. En los últimos años se ha presentado un incremento en la cantidad de compañías colombianas que reportan sobre su desempeño social y ambiental. Según la base de datos de GRI ', de 27 reportes en 2009, Colombia pasó a 182 en 2017. Por otra parte, y según el informe de la Encuesta

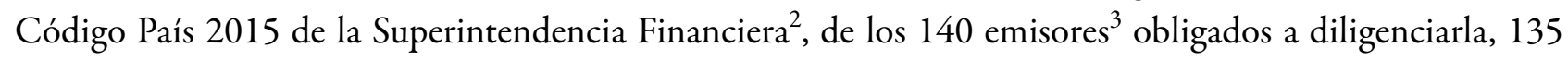
empresas reportaron información.

Las empresas familiares han venido implementando la revelación de información a partir del uso de informes de sostenibilidad. Sin embargo, los estudios se han enfocado en el GC a partir de la presencia de miembros de la familia en la junta, sin avanzar en la comparación de aspectos como contar con miembros independientes o contar con un comité de asuntos relacionado con ética, sostenibilidad y gobierno (Hsueh, 2018). Las comparaciones entre empresas familiares y no familiares para evaluar la capacidad de reportar información no financiera y su relación con la estructura de las juntas directivas han sido poco investigadas en la literatura (Iyer \& Lulseged, 2013), particularmente en economías emergentes. 
Si bien las diferentes guías y manuales sobre reporte de información han promovido en las empresas la estructuración de una junta directiva con miembros independientes y comités que incentiven una mayor transparencia (Fuentes, García-Sanchez, \& Lozano, 2017), y existen modelos teóricos que explican la relación entre la estructura de la junta directiva con el desempeño medioambiental y social (Shaukat, Qiu, \& Trojanowski, 2016), son escasos los estudios sobre la relación entre la estructura de la junta directiva con el reporte no financiero, centrado en los indicadores de sostenibilidad del GRI (Herda, Taylor, \& Winterbotham, 2012).

Los estudios sobre gobierno corporativo - GC- se han basado en la dicotomía entre la teoría de la dependencia de recursos -TDR- (Pfeffer, \& Salancik, 1978) y la teoría de la agencia -TA- (Jensen \& Meckling, 1976), sobre la que han surgido modelos y propuestas integradoras como las de Zahra y Pearce (1989), la de Hillman y Dalziel (2003) o más recientemente la de Pugliese, Minichilli y Zattoni (2014), que coinciden en que existen factores contingentes que hacen que los atributos de las juntas directivas incidan en el desempeño (Orozco et al., 2018). Los factores en el nivel de las firmas que son más sensibles a las características el GC son la estructura de propiedad y la reputación corporativa. Estas características inciden en la tendencia a revelar información, especialmente no financiera, a los diversos grupos de interés, ya que se espera que las empresas familiares busquen legitimación en un entorno en el que se compite por la reputación corporativa.

En este sentido, el objetivo de esta investigación es evaluar la relación de la estructura de la junta directiva en términos del tamaño, la independencia y la existencia de comités en asuntos de RSC con la revelación de información no financiera en los informes de sostenibilidad GRI de las empresas colombianas con mayor reputación en Merco Colombia comparando empresas familiares y no familiares.

El desarrollo del artículo está estructurado en cuatro secciones, aparte de esta introducción; la primera sección presenta el marco teórico y la revisión de literatura. En la segunda se define la metodología de que guio la investigación. En la tercera sección se presentan los resultados y se finaliza con una sección de discusión y conclusiones en la que se exponen las limitaciones y la investigación futura.

\section{Enfoque teórico y revisión de la literatura}

\section{La junta directiva como instancia estratégica para el reporte de RSC}

\section{Tamaño de la junta}

La junta directiva, como órgano central en el sistema de GC, tiene dos grandes tareas a cargo: el control de la administración y la toma de decisiones estratégicas. En los estudios sobre GC desde la Teoría de la Agencia -TA- (Jensen \& Meckling, 1976) se entiende que las juntas directivas pequeñas tienen mejor capacidad de ejercer control sobre la administración y tomar decisiones en el plano financiero. Estas juntas logran tener mejor consenso y facilitan el proceso de toma de decisiones estratégicas (Jensen, 1993). Sin embargo, desde la Teoría de Dependencia de Recursos -TDR- (Pfeffer, 1972; Pfeffer \& Salancik, 1978), se entiende que las juntas directivas que tienen mayor tamaño son capaces de atender gran diversidad de temas más allá de los asuntos financieros, como los aspectos sociales y medioambientales. Asimismo, entre más directores se tiene la posibilidad de ajustarse al entorno institucional de valores, normas y cogniciones mejorando la legitimidad organizacional.

Cuadrado, García y Martínez (2015) realizaron un estudio con una muestra de 1.043 empresas de 12 países desarrollados, y encontraron que el número de miembros de junta tiene una relación directa con las prácticas en RSC, no obstante, al superar los 19 miembros las prácticas de RSC se reducen posiblemente debido a los problemas de consenso y focalización de directrices. En esa vía el estudio de Arayssi, Dah, y Jizi (2016) sobre 
las empresas listadas en el Financial Times Stock Exchange para los años 2007 a 2012 encontraron que "Our results also recommend reduced board size helps firms reveal more information” (Arayssi et al., 2016, p. 385).

De otra parte, De Villiers, Naiker y Van Staden (2011) apoyados en la TDR dan evidencia con la información de la KLD database ${ }^{4}$ para una muestra de 1.216 firmas de Estados Unidos entre 2003 y 2004 que a mayor tamaño de la junta mayor el desempeño medioambiental. Chang et al. (2017) estudiando 780 empresas entre 2003 y 2005 en Corea, así como Huafang y Jianguo (2007) para 559 empresas listadas en China, encontraron que una junta grande está relacionada con una mejor divulgación de información social y medioambiental. De la misma forma estudios como el de Shamil et al. (2014) sobre 148 compañías listadas en Siri Lanka, el de Janggu et al. (2014) sobre 818 empresas listadas en Malasia, el trabajo de Al-Shaer y Zaman (2016) sobre 333 empresas en Reino Unido y el trabajo de Jizi et al., (2014) sobre 193 bancos en Estados Unidos encontraron que a mayor tamaño de las juntas mayor revelación de información en el GRI.

Dada la evidencia anterior se proponen las siguientes hipótesis:

H1a: A mayor tamaño de la junta directiva, mayor es la revelación y reporte de información sobre indicadores GRI ambientales.

H1b: A mayor tamaño de la junta directiva, mayor es la revelación y reporte de información sobre indicadores GRI sociales.

\section{Independencia de la junta}

En este punto tanto la TDR como la TA concuerdan en la importancia de contar con miembros independientes en las juntas directivas. En el marco de la TDR los miembros independientes son un recurso único que conecta a la organización con sus partes interesadas externas (Pfeffer, 1972) y contribuyen a mejorar las decisiones porque suman conocimientos y experiencias y "the outside board members act as arbiters in disagreements" (Fama \& Jensen, 1983, p. 315) mejorando la gobernabilidad y el control de las juntas y su relación con la administración, así como la reputación y legitimidad de la organización.

Si bien estudios como el de Cuadrado et al. (2015) concluyen que la presencia de miembros independientes tiene un impacto positivo en las prácticas de RSC, existe un punto, alrededor de cuatro miembros independientes, donde incluir un miembro adicional podría reducir las prácticas de RSC, la evidencia sugiere que entre más independientes mejor el desempeño. Como sugieren Nieto, Fernández y Cabeza (2012), "la independencia del consejo de administración tiene un efecto positivo y significativo sobre la realización de actividades de naturaleza social" (Nieto et al., 2012, p. 324). Al respecto, el estudio de De Villers et al. (2011) muestra una relación positiva entre la tenencia de miembros independientes y el desempeño medioambiental de las grandes corporaciones norteamericanas.

Sobre las prácticas de revelación social y ambiental, adicional a los estudios ya referenciados de Chang et al. (2017) y Huafang y Jianguo (2007) en Asia; Lorenzo, Sánchez, \& Gallego (2009) para 96 empresas no financieras de España, Fuentes et al. (2017) para 686 empresas listadas en la bolsa de valores de Madrid, Taylor y Winterbotham (2012) para 500 empresas en Estados Unidos; Post, Rahman y Rubow (2011) para 78 empresas del Fortune 1000, Herda et al. (2012) y el sistema bancario en Estados Unidos y Kiliç, Kuzey y Uyar (2015) para el caso turco encuentran una relación positiva entre el número de miembros independientes y la revelación de información empresarial social y ambiental. Finalmente, los estudios antes referenciados de De Villiers et al. (2011) y confirman que a mayor número de miembros independientes en las juntas, mejor es el desempeño y la revelación de información medioambiental.

De acá que se plantean las siguientes hipótesis:

H2a: A mayor número de miembros independientes en la junta directiva, mayor es la revelación y reporte de información sobre indicadores GRI ambientales. 
H2b: A mayor número de miembros independientes en la junta directiva, mayor es la revelación y reporte de información sobre indicadores GRI sociales.

\section{Comités de ética, gobierno o de sostenibilidad}

Otro de los componentes importantes en la junta directiva son sus comités, los cuales abordan diferentes asuntos como riesgos, auditoría, nominaciones, remuneraciones, asuntos financieros, entre otros. Desde la teoría de los stakeholders la toma de decisiones a partir del debate sobre los resultados y beneficiarios de las acciones organizacionales es un asunto diferencial en el desempeño corporativo (Freeman, 1999). Uno de los elementos centrales en la composición de las juntas directivas es la especialización en la toma de decisiones para diversos asuntos, entre ellos los que tienen que ver con la RSC. Los comités promueven mejores procesos de relacionamiento con los stakeholders, pues permite manejar los problemas de legitimidad de asuntos como los de RSC de una forma estratégica (Arnone et al., 2011), proteger a los grupos de interés, crear valor económico y social, así como reducir la corrupción (Gennari \& Salvioni, 2019). De hecho, Eccles, Iannouy y Serafeim (2014) muestran que las firmas más sustentables en los Estados Unidos cuentan con un comité para asuntos de RSC en sus juntas directivas.

En la revisión de Dienes, Sassen y Fischer (2016) encuentran que contar con comités para temas de sostenibilidad incide positivamente en la revelación de información sobre el desempeño medioambiental. $\mathrm{O}$ investigaciones como la de Fuente et al. (2017) que analiza la información sobre sostenibilidad en el GRI; Michelon y Parbonetti (2012) para las empresas listadas en el índice de sostenibilidad del Dow Jones en el año 2003; Amran, Lee, \& Devi (2014) para las empresas asiáticas y de Australia, Danvila del Valle, Diez y Lópezde-Foronda (2013) para una muestra de 469 firmas multinacionales listadas en el índice de sostenibilidad del Dow Jones y a su vez incluidas en el índice Eurostox 500, encuentran que existe una relación positiva entre contar con un comité que maneja asuntos ética, responsabilidad social corporativa y gobierno con la revelación de información sobre sostenibilidad en el GRI.

Por lo tanto, contar con un comité que trate los asuntos de ética, de GC o de sostenibilidad en la junta es clave para que las firmas le apuesten a la RSC y la sostenibilidad y a su vez, tiendan a revelar al mercado más información no financiera, en este caso ambiental y social. Por lo tanto se plantean las siguientes hipótesis:

H3a: La presencia de un comité de ética, de GC o de sostenibilidad en la junta directiva, promueve una mayor revelación y reporte de información sobre indicadores GRI ambientales.

H3b: La presencia de un comité de ética, de GC o de sostenibilidad en la junta directiva, promueve una mayor revelación y reporte de información sobre indicadores GRI sociales.

\section{Estructura de propiedad y reputación corporativa}

De acuerdo con la literatura, se considera que una empresa es familiar si está controlada económica, financiera o administrativamente por una o más familias de manera sustancial (Klein, 2000). Para las familias propietarias, las empresas son una forma de extensión de sí mismas. En este sentido las empresas familiares son sensibles al riesgo reputacional y esto explica la existencia de muchos esfuerzos por mantener e incrementar su buen nombre e imagen.

Si bien estudios como el Iyer y Lulseged (2013) indican que no hay diferencia estadísticamente significativa entre empresas familiares y no familiares en la probabilidad de presentar informes de sostenibilidad, Gavana et al. (2017a; 2017b) encuentran que hay una relación positiva entre las empresas familiares y la divulgación de información no financiera. Esto se explica porque las empresas familiares son más sensibles a la exposición de los medios y porque los beneficios que trae la divulgación de información de RSC, reducción del costo de capital, la prevención de la regulación o el litigio y, por tanto, un aumento de su reputación. La revisión de 
literatura de Sageder, Mitter y Feldbauer (2018) confirma que en las empresas familiares existe una relación positiva entre la reputación corporativa y el éxito en metas no financieras que se comunican a través de reportes de sostenibilidad.

Finalmente es de esperarse que las empresas con mejor reputación corporativa tiendan a ser más transparentes y a revelar más información. Michelon (2011) realiza un estudio a partir de la información de GRI para 57 firmas de Dow Jones Sustainability Index (DJSI) comparado con una muestra pareada de 57 firmas del Dow Jones Global Index para el 2003 en países de Europa y Estados Unidos para probar que "company's reputation is a determinant of sustainability disclosure" (Michelon, 2011, p. 79). Para el caso colombiano se sabe que las firmas familiares no son las mejor posicionadas en el escalafón de Merco sobre reputación corporativa. Se encontró que las empresas familiares, en general, "do not tend to have large boards, are not listed in stock markets and are not at the top of reputed firms in Colombia" (Orozco et al., 2018, p. 192). De acuerdo con lo anterior se esperaría que:

H4a: Las empresas familiares presentan una mayor revelación y reporte de indicadores ambientales.

H4b: Las empresas familiares presentan una mayor revelación y reporte de indicadores sociales.

H4c: Las empresas con mayor reputación corporativa no son familiares y presentan una mayor revelación y reporte de indicadores ambientales y sociales.

\section{Metodología}

\section{Muestra}

Se seleccionó una muestra de 115 empresas incluidas en el escalafón Merco de reputación corporativa para Colombia ${ }^{5}$ entre los años 2008 y 2014 . Se obtuvo el informe de sostenibilidad GRI a partir de la Sustainability Disclosure Database para 88 firmas con la versión G4 del periodo 2014. Para 27 casos restantes se obtuvo la información de los informes corporativos publicados por las firmas en sus páginas web que utilizan el estándar GRI. Estas compañías se incluyen en la muestra dado que son muy representativas del parque empresarial colombiano y reiteradamente clasifican en Merco. Para la información de juntas directivas, además de los informes corporativos, se consultó la encuesta Código País de las compañías que la publicaron dentro de sus páginas web o en la página de la Superintendencia de Sociedades en Colombia ${ }^{6}$.

Se realiza un análisis multivariado de correlaciones de Spearman y regresiones con el método de mínimos cuadrados ordinarios. Asimismo, se utilizó la técnica de análisis de clúster, para clasificar las empresas de acuerdo con la diferencia de medias en las variables de análisis y comparar los grupos donde hay mayor y menor revelación ambiental y social. Finalmente, se presenta la prueba F para diferencia de medias con el fin de evaluar la hipótesis nula de que las medias entre los grupos son estadísticamente diferentes con un pvalor del $10 \%$.

\section{Variables dependientes}

- Número de indicadores medioambientales reportados - Ambiente GRI: es la sumatoria de la cantidad de indicadores ambientales GRI en su versión G4 reportados para el periodo 2014 y que corresponden a: G4-EN3 consumo energético interno, G4-EN6 porcentaje de reducción del consumo energético, G4-EN8 captación de agua según fuente, G4-EN10 porcentaje de agua reciclada / reutilizada, G4-EN15 emisiones directas alcance 1, G4-EN16 emisiones indirectas alcance 2, G4-EN19 porcentaje de reducción de emisiones de gases de efecto invernadero y G4-EN23 peso total de residuos. 
- Número de indicadores sociales reportados - Social GRI: es la sumatoria de la cantidad de indicadores sociales GRI en su versión G4 reportados para el periodo 2014 y que corresponden a G4-LA1 número total directo de mujeres al cierre 2014, G4-LA6 tasa de accidentalidad, G4-LA9 promedio horas de formación por empleado, G4-HR2 número de horas de formación en derechos humanos, G4-HR2 porcentaje de empleados capacitados, G4-SO8 valor monetario de multas significativas (sociales), G4-PR2 número de incidentes por incumplimiento en salud y seguridad clientes y G4-PR9 valor monetario de multas significativas (clientes).

\section{Variables independientes}

- Tamaño de Junta - TJD: es el número de miembros de junta directiva (incluidos principales y suplentes).

- Número de miembros independientes de la junta directiva - Indep. Es el número de miembros independientes declarado en el indicador G4-38. En concordancia con los criterios establecidos en el parágrafo 2 del artículo 44 de la Ley 964 de $2005^{7}$.

- Comité de ética, de GC o de sostenibilidad - Comité É/GC/S. Variable dicotómica, que tomará el valor de 1 si en la junta directiva existe un comité que trate los asuntos de ética, sostenibilidad y gobierno corporativo, de acuerdo con la información del indicador G4-38 del estándar GRI en su versión G4, y 0 en el caso contrario.

\section{Variables de control}

- Empresas familiares - Fam. Variable dicotómica. Se verificó en cada empresa la estructura de propiedad y se evaluó si hay un controlante mayoritario que no responde a un inversionista institucional. A partir del GRI G4-7 del estándar GRI en su versión G4. Toma el valor de 1 si es familiar y 0 en caso contrario.

- Reputación corporativa - Merco. Es la posición promedio de las empresas en el ranking Merco en Colombia, entre los años 2008 y 2014 invertida con el fin de mantener la lógica de las regresiones que analiza si hay o no un aumento sistemático de las variables.

\section{Resultados}

\section{Estadísticos descriptivos de la muestra seleccionada}

Del total de las 115 empresas objeto de estudio, el 59\% (68) son empresas de origen colombiano, mientras que el 41\% (47) son de origen internacional con operación en Colombia. De las 115 empresas, el 77\% (88) publicaron sus indicadores en sostenibilidad siguiendo los lineamientos GRI G4 para el año 2014. Asimismo, el 94\% (108 empresas) hacen pública su junta directiva, frente a un 6\% (7) que no lo hacen. De las 108 empresas que publican su junta directiva, el $8 \%$ ( 9 empresas) no publican si tienen miembros independientes. Por otra parte, del total de empresas observadas (115), el 75\% (86) publicaron sus comités de junta directiva y el 25\% (29) no lo hicieron.

En la tabla 1 se presentan los estadísticos descriptivos para las variables dependientes, así como para el tamaño y el número de miembros independientes en las juntas, y las frecuencias para empresas con comité de É/GC/S o si son o no familiares. Se observa que la media de indicadores GRI G4 reportados es de cuatro indicadores, en aquellas empresas que publicaron sus informes de sostenibilidad en 2014. Por otra parte, el 
tamaño de la junta directiva (incluidos principales y suplentes) llega a una media de 10 miembros, donde cuatro de ellos ostentan la calidad de independientes. Se encuentran 41 empresas con comités de asuntos de GC, ética o sostenibilidad. Finalmente se encuentran 51 empresas familiares y 64 no familiares.

TABLA 1

Estadísticos descriptivos

\begin{tabular}{|l|c|c|c|c|c|c|c|}
\hline \multicolumn{1}{|c|}{ Variable } & n & Min & Máx & Media & Total & Si & No \\
\hline Ambiente GRI & 115 & 0 & 8 & 4,41 & & & \\
\hline Social GRI & 113 & 0 & 10 & 4,35 & & & \\
\hline TJD & 115 & 0 & 42 & 9,95 & & & \\
\hline Indep & 115 & 0 & 13 & 3,95 & & & \\
\hline Comité É/GC/S & & & & & 115 & 41 & 74 \\
\hline Fam & & & & & 115 & 51 & 64 \\
\hline
\end{tabular}

Fuente: elaboración propia.

\section{Análisis de correlaciones}

Como siguiente paso se llevó a cabo un análisis de correlaciones con el método de Spearman ya que hay dos variables dicotómicas. En la tabla 2 se puede observar que el número de indicadores sociales y ambientales tiene relación con el tamaño de la junta, la independencia de sus miembros, el comité de sostenibilidad, la posición en el escalafón de reputación corporativa y una relación negativa con las empresas familiares. Esto indica que las firmas familiares podrían estar reportando menos que las empresas de propiedad anónima. Como es de esperarse, el tamaño de la junta tiene una alta correlación con la tenencia de miembros independientes.

Se evidencia una relación positiva moderada entre la existencia de un comité de ética, de gobierno o sostenibilidad con el reporte de información ambiental y una relación positiva débil con el reporte de información social. Por otra parte, se observa que la existencia de un comité de ética, de GC o de sostenibilidad tiene una relación positiva con el número de miembros independientes. Se encontró también que existe una correlación positiva y significativa entre la cantidad de miembros independientes y la existencia de un comité de la junta para asuntos de RSC. Se encuentra que la reputación se relaciona con el tamaño de la junta, la independencia y la tenencia de comités para RSC en las juntas. Finalmente, las empresas familiares tienen una correlación negativa con las características de las juntas directivas y la reputación corporativa. 
TABLA 2

Correlaciones

\begin{tabular}{|c|c|c|c|c|c|c|c|c|c|}
\hline & & & $\begin{array}{c}\text { Ambiente } \\
\text { GRI }\end{array}$ & $\begin{array}{c}\text { Social } \\
\text { GRI }\end{array}$ & TJD & Indep & $\begin{array}{l}\text { Comité } \\
\text { ÉGCS }\end{array}$ & Merco & Fam \\
\hline \multirow{21}{*}{$\begin{array}{l}\text { Rho de } \\
\text { Spearman }\end{array}$} & \multirow{3}{*}{$\begin{array}{l}\text { Ambiente } \\
\text { GRI }\end{array}$} & $\begin{array}{l}\text { Coeficiente de } \\
\text { correlación }\end{array}$ & 1,000 & $0,711^{* *}$ & $0,328^{*}$ & $0,497^{*}$ & $0,355^{*}$ & $0,262^{*}$ & $-0,296^{*}$ \\
\hline & & Sig. (bilateral) & & 0,000 & 0,000 & 0,000 & 0,000 & 0,005 & 0,001 \\
\hline & & $\mathrm{N}$ & 115 & 113 & 115 & 115 & 115 & 115 & 115 \\
\hline & \multirow{3}{*}{$\begin{array}{l}\text { Social } \\
\text { GRI }\end{array}$} & $\begin{array}{l}\text { Coeficiente de } \\
\text { correlación }\end{array}$ & $0,711^{*}$ & 1,000 & $0,270^{*}$ & $0,445^{*}$ & $0,354^{* *}$ & $0,297^{*}$ & $-0,237^{\circ}$ \\
\hline & & Sig. (bilateral) & 0,000 & & 0,004 & 0,000 & 0,000 & 0,001 & 0,012 \\
\hline & & $\mathrm{N}$ & 113 & 113 & 113 & 113 & 113 & 113 & 113 \\
\hline & \multirow{3}{*}{ TJD } & $\begin{array}{l}\text { Coeficiente de } \\
\text { correlación }\end{array}$ & $0,328^{* *}$ & $0,270^{*}$ & 1,000 & $0,586^{*}$ & 0,113 & 0,097 & $-0,165$ \\
\hline & & Sig (bilateral) & 0,000 & 0,004 & & 0,000 & 0,230 & 0,301 & 0,077 \\
\hline & & $\mathrm{N}$ & 115 & 113 & 115 & 115 & 115 & 115 & 115 \\
\hline & \multirow{3}{*}{ Indep } & $\begin{array}{l}\text { Coeficiente de } \\
\text { correlación }\end{array}$ & $0,497^{* *}$ & $0,445^{* *}$ & $0,586^{*}$ & 1,000 & $0,304^{* *}$ & $0,275^{*}$ & $-0,240^{*-}$ \\
\hline & & Sig. (bilateral) & 0,000 & 0,000 & 0,000 & & 0,001 & 0,003 & 0,010 \\
\hline & & $\mathrm{N}$ & 115 & 113 & 115 & 115 & 115 & 115 & 115 \\
\hline & \multirow{3}{*}{$\begin{array}{l}\text { Comité } \\
\text { É/GC/S }\end{array}$} & $\begin{array}{l}\text { Coeficiente de } \\
\text { correlación }\end{array}$ & $0,355^{*}$ & $0,354^{* *}$ & 0,113 & $0,304^{*}$ & 1,000 & 0,177 & $-0,299^{*}$ \\
\hline & & Sig. (bilateral) & 0,000 & 0,000 & 0,230 & 0,001 & & 0,059 & 0,001 \\
\hline & & $\mathrm{N}$ & 115 & 113 & 115 & 115 & 115 & 115 & 115 \\
\hline & \multirow{3}{*}{ Merco } & $\begin{array}{l}\text { Coeficiente de } \\
\text { correlación }\end{array}$ & $0,262^{* *}$ & $0,297^{*}$ & 0,097 & $0,275^{*}$ & 0,177 & 1,000 & $-0,186^{\circ}$ \\
\hline & & Sig. (bilateral) & 0,005 & 0,001 & 0,301 & 0,003 & 0,059 & & 0,046 \\
\hline & & $\mathrm{N}$ & 115 & 113 & 115 & 115 & 115 & 115 & 115 \\
\hline & \multirow{3}{*}{ Fam } & $\begin{array}{l}\text { Coeficiente de } \\
\text { correlación }\end{array}$ & $-0,296^{*}$ & $-0,237^{\circ}$ & $-0,165$ & $-0,240^{*}$ & $-0,299^{*}$ & $-0,186^{\circ}$ & 1,000 \\
\hline & & Sig. (bilateral) & 0,001 & 0,012 & 0,077 & 0,010 & 0,001 & 0,046 & \\
\hline & & $\mathrm{N}$ & 115 & 113 & 115 & 115 & 115 & 115 & 115 \\
\hline
\end{tabular}

\section{Análisis de regresiones}

Como se observa en las tablas 3 y 4, el primer modelo se aplicó a los indicadores ambientales y el segundo a los sociales. Para el primer modelo, con un $\mathrm{R}$ cuadrado de 0.35 y para el segundo con $\mathrm{R}$ cuadrado de 0.30 , se concluye que no se rechazan las hipótesis $\mathrm{H} 1 \mathrm{a}, \mathrm{H} 2 \mathrm{a}, \mathrm{H} 3 \mathrm{a}, \mathrm{H} 1 \mathrm{~b}, \mathrm{H} 2 \mathrm{~b}$ y H3b, y se puede afirmar, con una relación estadísticamente significativa al 10\%, que el número de miembros independientes de la junta, tamaño de la junta y la existencia de un comité de ética, gobierno o sostenibilidad tienen una relación positiva con el reporte de indicadores ambientales y sociales. Asimismo, se observa una asociación positiva y significativa entre la reputación corporativa con el reporte de indicadores, y las empresas familiares no presentan una relación significativa con la revelación de información ambiental y social, con lo que se rechazan la hipótesis H4a y H4b. Por tal razón no podemos afirmar que las empresas familiares presentan una mayor revelación y reporte de indicadores sociales que las que no lo son. Finalmente rechazamos $\mathrm{H} 4 \mathrm{c}$ ya que las empresas familiares no son las de mayor reputación de acuerdo con la tabla 1 y tampoco son las que más revelan información.

TABLA 3

Modelo de regresión con reporte de indicadores ambientales

\begin{tabular}{|c|c|c|c|c|c|c|}
\hline \multicolumn{7}{|c|}{ Variable dependiente: Ambiente GRI } \\
\hline \multirow{2}{*}{\multicolumn{2}{|c|}{ Modelo }} & \multicolumn{2}{|c|}{$\begin{array}{l}\text { Coeficientes no } \\
\text { estandarizados }\end{array}$} & \multirow{3}{*}{$\begin{array}{c}\begin{array}{c}\text { Coeficientes } \\
\text { estandarizados }\end{array} \\
\text { Beta } \\
\end{array}$} & \multirow{3}{*}{$\begin{array}{c}\mathrm{t} \\
2,067 \\
\end{array}$} & \multirow{3}{*}{$\begin{array}{l}\text { Sig. } \\
0,041\end{array}$} \\
\hline & & \multirow{2}{*}{$\begin{array}{c}\text { B } \\
1,486\end{array}$} & \multirow{2}{*}{$\begin{array}{c}\text { Desv. Error } \\
0,719\end{array}$} & & & \\
\hline 1 & (Constante) & & & & & \\
\hline & TJD & 0,092 & 0,053 & 0,151 & 1,729 & 0,087 \\
\hline & Indep & 0,257 & 0,076 & 0,302 & 3,367 & 0,001 \\
\hline & Comité É/GC/S & 1,394 & 0,526 & 0,218 & 2,650 & 0,009 \\
\hline & Merco & 0,014 & 0,007 & 0,146 & 1,829 & 0,070 \\
\hline & Fam & $-0,655$ & 0,510 & $-0,106$ & $-1,284$ & 0,202 \\
\hline
\end{tabular}

Fuente: elaboración propia. 
TABLA 4

Modelo de regresión con reporte de indicadores sociales

\begin{tabular}{|c|c|c|c|c|c|c|}
\hline \multicolumn{7}{|c|}{ Variable dependiente: Social GRI } \\
\hline \multirow{2}{*}{\multicolumn{2}{|c|}{ Modelo }} & \multicolumn{2}{|c|}{$\begin{array}{l}\text { Coeficientes no } \\
\text { estandarizados }\end{array}$} & \multirow{2}{*}{$\begin{array}{c}\text { Coeficientes } \\
\text { estandarizados }\end{array}$} & \multirow[t]{2}{*}{ t } & \multirow[t]{2}{*}{ Sig. } \\
\hline & & B & Desv. Error & & & \\
\hline \multirow[t]{6}{*}{1} & (Constante) & 1,202 & 0,731 & & 1,645 & 0,103 \\
\hline & TJD & 0,113 & 0,054 & 0,190 & 2,086 & 0,039 \\
\hline & Indep & 0,165 & 0,077 & 0,202 & 2,156 & 0,033 \\
\hline & Comité É/GC/S & 1,524 & 0,528 & 0,248 & 2,888 & 0,005 \\
\hline & Merco & 0,016 & 0,007 & 0,179 & 2,148 & 0,034 \\
\hline & Fam & $-0,317$ & 0,514 & $-0,053$ & $-0,617$ & 0,538 \\
\hline
\end{tabular}

Fuente: elaboración propia.

\section{Análisis de clúster}

$\mathrm{Al}$ aplicar el análisis de clúster se identifican dos grupos: el primero con 56 empresas y el segundo con 55 . El primer clúster está compuesto principalmente por empresas familiares que muestran en promedio menores valores para las variables analizadas que el segundo clúster como se muestra en la tabla 5 . La diferencia de las medias entre los dos clústers es estadísticamente válida al 10\%, como se observa en la Anova y la prueba F de la tabla 6 para todas las variables excepto la familiar que, pese a tener claramente definidos los centroides entre los clústers, la diferencia de medias tiene un $\mathrm{p}$-valor $>=16 \%$. Esto sugiere que los resultados son indicativos de una tendencia entre empresas familiares y no familiares, pero que gracias a los resultados previos en los modelos de regresión, se puede establecer que las empresas familiares tienden a reportar menos indicadores que las firmas con otra estructura de propiedad.

TABLA 5

Clúster variables

\begin{tabular}{|l|r|r|}
\hline \multirow{2}{*}{ Centros de clústeres finales } & \multicolumn{2}{|c|}{ Clúster } \\
\cline { 2 - 3 } & 1 & 2 \\
\hline Ambiente GRI & 4 & 5 \\
\hline Social GRI & 3,55 & 5,14 \\
\hline TJD & 9 & 11 \\
\hline Indep & 3 & 5 \\
\hline Comité É/GC/S & 0 & 0 \\
\hline Merco & 30 & 87 \\
\hline Fam & 1 & 0 \\
\hline
\end{tabular}

Fuente: elaboración propia.

TABLA 6

ANOVA

\begin{tabular}{|c|c|c|c|c|c|c|}
\hline & \multicolumn{2}{|c|}{ Clúster } & \multicolumn{2}{|c|}{ Error } & \multirow[b]{2}{*}{ F } & \multirow[b]{2}{*}{ Sig. } \\
\hline & $\begin{array}{c}\text { Media } \\
\text { cuadrática }\end{array}$ & gl & $\begin{array}{c}\text { Media } \\
\text { cuadrática }\end{array}$ & gl & & \\
\hline Ambiente GRI & 89,413 & 1 & 8,566 & 111 & 10,438 & 0,002 \\
\hline Social GRI & 71,124 & 1 & 8,241 & 111 & 8,631 & 0,004 \\
\hline TJD & 131,651 & 1 & 24,128 & 111 & 5,456 & 0,021 \\
\hline Indep & 51,119 & 1 & 12,765 & 111 & 4,005 & 0,048 \\
\hline Comité É/GC/S & 0,660 & 1 & 0,229 & 111 & 2,878 & 0,093 \\
\hline Merco & 91719,567 & 1 & 280,045 & 111 & 327,517 & 0,000 \\
\hline Fam & 0,489 & 1 & 0,246 & 111 & 1,991 & 0,161 \\
\hline
\end{tabular}

Fuente: elaboración propia. 


\section{Discusión y conclusiones}

Tanto la TDR como la TA ponen énfasis en la importancia que tiene la estructura de gobierno corporativo y la composición de las juntas directivas para mejorar las actividades de control y toma de decisiones en la definición de estrategias y políticas corporativas. Una de las formas en las que incide la estructura de las juntas directivas se encuentra en el esfuerzo por desarrollar actividades de RSC y la revelación de información no financiera. En el marco de los modelos integradores entre la TA y la TDR como el de Pugliese et al. (2014), se determinan variables contingentes que inciden en la forma en que una u otra característica del GC se relaciona con el desempeño como lo es la estructura de propiedad comparando firmas familiares y no familiares.

En esta investigación extendemos los estudios previos sobre la relación entre el GC y la revelación de información social y medioambiental a partir de la discusión de dos variables contingentes: la estructura de propiedad diferenciando firmas familiares y no familiares, así como la reputación corporativa. Para el caso colombiano, los resultados sugieren que un mayor tamaño de la junta directiva, con mayor número de miembros independientes y la existencia de un comité para tratar asuntos de ética, gobierno o sostenibilidad, inciden en una mayor revelación de información no financiera, en este caso, de indicadores ambientales y sociales, y esto particularmente se observa en empresas de alta reputación según el monitor Merco pero que a su vez no son empresas familiares.

Cada vez existe un mayor consenso sobre la importancia del rol de la junta directiva en la revelación de información no financiera, particularmente en el ámbito social y medioambiental, e instrumentos como el GRI contribuyen a que las empresas puedan avanzar en la construcción de indicadores para darlos a conocer a sus grupos de interés. La evidencia sugiere que en la medida que las grandes corporaciones, con alta reputación tienen una mayor cantidad de temas en el marco de la RSC, acuden a estructurar juntas directivas de mayor tamaño, con más miembros independientes y comités para especializar el trabajo.

Es posible que las empresas familiares se encuentren en un proceso de estructuración y formalización de sus GC para tener mejores posibilidades de entrar en el círculo virtuoso de la reputación corporativa y la revelación de información no financiera. En este sentido una de las implicaciones para la práctica administrativa de esta investigación radica en la necesidad de apoyar a la mejor estructuración de los GC de las empresas familiares.

Estados Unidos o el Reino Unido se destacan por prácticas de buen GC, dada la rigurosidad de la ley y la cultura empresarial que ha buscado mejorar las prácticas de RSC y la revelación de las mismas. En Latinoamérica, acuerdos como el de la Alianza del Pacífico y su Mercado Integrado Latinoamericano MILA-, han impulsado un mayor ejercicio de revelación de información no financiera dentro de estándares del GRI, así como también la implementación de prácticas de buen gobierno corporativo.

Si bien en Colombia los avances legislativos, las iniciativas de diversos sectores y de entidades como la Superintendencia Financiera, la Superintendencia de Sociedades, las universidades, las cámaras de comercio y los gremios han incidido en que los empresarios busquen mejorar sus prácticas de RSC en el marco del buen GC, es necesario apoyar más a las empresas familiares. En la medida que estas empresas estructuren mejores GC, como se ha mostrado en estudios previos (Herrera-Echeverry et al., 2018; Orozco et al., 2018), se podrá avanzar en la transparencia y revelación de información en la promoción de prácticas de RSC y mejorar la reputación corporativa, tan importante en tiempos turbulentos como el de la pandemia del Covid-19.

Estos resultados son un aporte que invita al sector privado a reflexionar sobre las estructuras actuales de GC y su relación con el reporte de información no financiera. Sin embargo, este estudio tiene una serie de limitaciones. Al tener en cuenta compañías reconocidas y de gran tamaño, que no representan el común del parque empresarial colombiano, compuesto por pymes familiares, es importante leer los resultados en el marco de las firmas que se pueden mover en el marco del mercado de valores. Superar esta limitación requiere de investigación futura que indague sobre el comportamiento del desempeño sostenible en las pymes del país y la incidencia que tiene la estructura de su consejo de familia en dichos resultados. De igual forma, es 
importante incluir otras variables contingentes como el sector económico, tipo de reporte, aseguramiento a la información no financiera reportada y los sistemas de auditoría y control interno. Finalmente, una de las variables clave en la estructura del GC es la diversidad de los miembros. Particularmente la variable de género en la junta directiva ha sido reportada en estudios como los de Bear, Rahman y Post (2010), Boone, Casares, Karpoff y Raheja (2007), Dienes y Velte, (2016) y Carter, Simkins y Simpson (2003), quienes encontraron que el número de mujeres en la junta tienen una relación positiva con la revelación de información no financiera, tema que se sugiere para una futura investigación.

\section{Agradecimientos}

Este artículo parte del trabajo de grado de Alejandro Wilches Segovia, dirigido por el profesor Luis Antonio Orozco y evaluado por el profesor César Beltrán, quienes conjuntamente fortalecieron la investigación. Gracias a los comentarios de los pares de la revista Cuadernos de Administración. Este trabajo es dedicado a la Dra. María Claudia Romero, directora de la Maestría en Responsabilidad Social y Sostenibilidad de la Universidad Externado de Colombia por su invaluable gestión y aporte por la RSC.

\section{Referencias}

Al-Shaer, H., \& Zaman, M. (2016). Board gender diversity and sustainability reporting quality. Journal of Contemporary Accounting \& Economics, 12(3), 210-222. https://doi.org/10.1016/j.jcae.2016.09.001

Amran, A., Lee, S. P., \& Devi, S. S. (2014). The influence of governance structure and strategic corporate social responsibility toward sustainability reporting quality. Business Strategy and the Environment, 23(4), 217-235. h ttps://doi.org/10.1002/bse.1767

Arayssi, M., Dah, M., \& Jizi, M. (2016). Women on boards, sustainability reporting and firm performance. Sustainability Accounting, Management and Policy Journal, 7(3), 376-401. https://doi.org/10.1108/SAMPJ-0 7-2015-0055

Arnone, L., Ferauge, P., Geerts, A., \& Pozniak, L. (2011). Corporate social responsibility: Internet as communication tool towards stakeholders. Journal of Modern Accounting and Auditing, 7(7), 697. https://www.semanticscholar.org/paper/Corporate-Social-Responsibility\%EF\%BC\%9A-Internet-as-To ol-Laurent-Amone/e6b4f08bfde515916f367ab69c95c57ee6fc5a41?p2df

Bear, S., Rahman, N., \& Post, C. (2010). The impact of board diversity and gender composition on Corporate Social Responsibility and firm reputation. Journal of Business Ethics, 97(2), 207-221. https://doi.org/10.1007/s1055 1-010-0505-2

Boone, A. L., Casares Field, L., Karpoff, J. M., \& Raheja, C. G. (2007). The determinants of corporate board size and composition: An empirical analysis. Journal of Financial Economics, 85(1), 66-101. https://doi.org/10.1016/j. jfineco.2006.05.004

Carter, D., Simkins, B., \& Simpson, W. (2003). Corporate Governance, Board Diversity, and Firm Value. The Financial Review, 38, 33-53. https://doi.org/10.1111/1540-6288.00034

Chang, Y. K., Oh, W., Park, J. H., \& Jang, M. G. (2017). Exploring the relationship between board characteristics and CSR: Empirical evidence from Korea. Journal of Business Ethics, 140, 225-242. https://doi.org/10.1007/s105 51-015-2651-z

Cuadrado, B., García, R., \& Martínez, J. (2015). Efecto de la composición del consejo de administración en las prácticas de responsabilidad social corporativa. Revista de Contabilidad, 18(1), 20-31. http://dx.doi.org/10.1016/j.rcsar .2014 .02 .003

Danvila del Valle, I., Diez Esteban, J. M., \& López-de-Foronda, O. (2013). Corporate Social Responsibility and Sustainability Committee Inside the Board. SSRN Working Paper Series, 34, 1-30. https://doi.org/http://dx.d oi.org/10.2139/ssrn.2260382 
De Villiers, C., Naiker, V., \& Van Staden, C. J. (2011). The effect of board characteristics on firm environmental performance. Journal of Management, 37(6), 1636-1663. https://doi.org/10.1177/0149206311411506

Dienes, D., \& Velte, P. (2016). The impact of supervisory board composition on CSR reporting. Evidence from the German two-tier system. Sustainability (Switzerland), 8(1), 1-20. https://doi.org/10.3390/su8010063

Dienes, D., Sassen, R., \& Fischer, J. (2016). What are the drivers of sustainability reporting? A systematic review. Sustainability Accounting, Management and Policy Journal, 7(2), 154-189. https://doi.org/10.1108/SAMPJ-0 8-2014-0050

Eccles, R. G., Iannou, I., \& Serafeim, G. (2014). The impact of corporate sustainability on organizational processes and performance. Management Science, 6, 2835-2857. https://doi.org/10.1287/mnsc.2014.1984

Fama, E. F., \& Jensen, M. C. (1983). Separation of ownership and control. The Journal of Law and Economics, 26(2), 301-325. https://doi.org/10.1086/467037

Fernandez-Feijoo, B., Romero, S., \& Ruiz-Blanco, S. (2014). Women on boards: Do they affect sustainability reporting? Corporate Social Responsibility and Environmental Management, 21(6), 351-364. https://doi.org/1 $0.1002 /$ csr. 1329

Freeman, E. (1999). Divergent stakeholder theory. Academy of Management Review, 24, 233-236. https://doi.org/10 $.5465 / \mathrm{amr} .1999 .1893932$

Fuentes, J. A., García-Sanchez, I. M., \& Lozano, M. B. (2017). The role of the board of directors in the adoption of GRI guidelines for the disclosure of CSR information. Journal of Cleaner Production, 141, 737-750. https://d oi.org/10.1016/j.jclepro.2016.09.155

Gavana, G., Gottardo, P., \& Moisello, A. M. (2017a). Sustainability reporting in family firms: A panel data analysis. Sustainability, 9(1), 38. https://doi.org/10.3390/su9010038

Gavana, G., Gottardo, P., \& Moisello, A. M. (2017b). The effect of equity and bond issues on sustainability disclosure. Family vs non-family Italian firms. Social Responsibility Journal, 13(1), 126-142. https://doi.org/10.1108/SRJ05-2016-0066

Gennari, F., \& Salvioni, D. M. (2019). CSR committees on boards: The impact of the external country level factors. Journal of Management and Governance, 23(3), 759-785. https://doi.org/10.1007/s10997-018-9442-8

Giannarakis, G. (2014). Corporate governance and financial characteristic effects on the extent of corporate social responsibility disclosure. Social Responsibility Journal, 10(4), 569-590. https://doi.org/10.1108/SRJ-02-20130008

Global Reporting Initiative (2016). Conjunto consolidado de Estándares GRI para la elaboración de informes de sostenibilidad. Global Reporting Initiative, 1-444. https://www.globalreporting.org/standards/gri-standards-tra nslations/gri-standards-spanish-translations-download-center/?g=7cbb8b53-11cd-4489-8de7-2bc7c2b86253

Global Reporting Initiative. (2013). Guía para la elaboración de memorias de sostenibilidad. Manual de aplicación. Versión G4. Recuperado de https://www.globalreporting.org/resourcelibrary/Spanish-G4-Part-Two.pdf

Herda, D. N., Taylor, M. E. \& Winterbotham, G. (2012). The effect of board independence on the sustainability reporting practices of large U. S. firms. Issues in Social and Environmental Accounting, 6(2), 178-197. https://d oi.org/10.22164/isea.v6i2.69

Herrera-Echeverri, H., Gaitan, S., Orozco, L A., \& Soto-Echeverry, N. (2018). Board Characteristics, Compliance, and Firm Performance: Empirical Findings from Colombia. Latin American Business Review, 19(3-4), 263-295. ht tps://doi.org/10.1080/10978526.2018.1540935

Hillman, A. J., \& Dalziel, T. (2003). Boards of directors and firm performance: Integrating agency and resource dependence perspectives. Academy of Management Review, 28(3), 383-396. https://doi.org/10.5465/amr.2003 .10196729

Hsueh, J. W. J. (2018). Governance structure and the credibility gap: Experimental evidence on family businesses' sustainability reporting. Journal of Business Ethics, 153(2), 547-568. https://doi.org/10.1007/s10551-016-340 $9-y$

Huafang, X., \& Jianguo, Y. (2007). Ownership structure, board composition and corporate voluntary disclosure. Managerial Auditing Journal. https://doi.org/10.1108/02686900710759406 
Iyer, V., \& Lulseged, A. (2013). Does family status impact US firms' sustainability reporting? Sustainability Accounting, Management and Policy Journal, 4(2), 163-189. https://doi.org/10.1108/SAMPJ-Nov-2011-0032

Janggu, T., Darus, F., Zain, M. M., \& Sawani, Y. (2014). Does good corporate governance lead to better sustainability reporting? An analysis using structural equation modeling. Procedia-Social and Behavioral Sciences, 145, 138-145. https://doi.org/10.1016/j.sbspro.2014.06.020

Jensen, M. (1993). The modern industrial revolution, exit, and the failure of internal control systems. Journal of Finance, 48(3), 831-880. https://doi.org/10.1111/j.1540-6261.1993.tb04022.x

Jensen, M. C., \& Meckling, W. H. (1976). Theory of the firm: Managerial behavior, agency costs and ownership structure. Journal of Financial Economics, 3, 305-360. https://doi.org/10.1016/0304-405X(76)90026-X

Jizi, M. I., Salama, A., Dixon, R., \& Stratling, R. (2014). Corporate governance and corporate social responsibility disclosure: Evidence from the US banking sector. Journal of Business Ethics, 125(4), 601-615. https://doi.org/ $10.1007 / \mathrm{s} 10551-013-1929-2$

Kiliç, M., Kuzey, C., \& Uyar, A. (2015). The impact of ownership and board structure on Corporate Social Responsibility (CSR) reporting in the Turkish banking industry. Corporate Governance, 15(3), 357-374. https ://doi.org/10.1108/CG-02-2014-0022

Klein, S. B. (2000). Family businesses in Germany: Significance and structure. Family Business Review, 13(3), 157-182. https://doi.org/10.1111/j.1741-6248.2000.00157.x

Lorenzo, J. M., Sánchez, I. M. G., \& Gallego-Álvarez, I. (2009). Características del consejo de administración e información en materia de responsabilidad social corporativa. Spanish Journal of Finance and Accounting/Revista Española de financiación y Contabilidad, 38(141), 107-135. https://doi.org/10.1080/02102412.2009.1077966 4

Michelon, G. (2011). Sustainability disclosure and reputation: A comparative study. Corporate Reputation Review, 14(2), 79-96. https://doi.org/10.1057/crr.2011.10

Michelon, G., \& Parbonetti, A. (2012). The effect of corporate governance on sustainability disclosure. Journal of Management and Governance, 16(3), 477-509. https://doi.org/10.1007/s10997-010-9160-3

Nieto, M., Fernández, R., \& Cabeza, L. (2012). La RSC en la relación entre composición del consejo y valor de mercado de la empresa. Papeles de Economía Española, (132), 315-328. https://dialnet.unirioja.es/servlet/extart?codigo $=3976069$

Orozco, L. A., Vargas, J., \& Galindo-Dorado, R. (2018). Trends on the relationship between board size and financial and reputational corporate performance: The Colombian case. European Journal of Management and Business Economics, 27(2), 183-197. https://doi.org/10.1108/EJMBE-02-2018-0029

Patten, D. M. (2002). The relation between environmental performance and environmental disclosure: a research note. Accounting, Organizations and Society, 27(8), 763-773. https://doi.org/10.1016/S0361-3682(02)00028-4

Patten, D. M. (2019). Seeking legitimacy. Sustainability Accounting, Management and Policy Journal. Vol. ahead-ofprint No. ahead-of-print. https://doi.org/10.1108/SAMPJ-12-2018-0332

Pfeffer, J. (1972). Size and composition of corporate boards of directors: The organization and its environment. Administrative Science Quarterly, 17(2), 218-228. https://doi.org/10.2307/2393956

Pfeffer, J., \& Salancik, G. R. (1978). The external control of organizations: A resource dependence perspective. Harper and Row. https://doi.org/10.2307/2231527

Post, C., Rahman, N., \& Rubow, E. (2011). Green governance: Boards of directors' composition and environmental corporate social responsibility. Business \& Society, 50(1), 189-223. https://doi.org/10.1177/00076503103946 42

Pugliese, A., Minichilli, A., \& Zattoni, A. (2014). Integrating agency and resource dependence theory: Firm profitability, industry regulation, and board task performance. Journal of Business Research, 67(6), 1189-1200. h ttps://doi.org/10.1016/j.jbusres.2013.05.003

Sageder, M., Mitter, C., \& Feldbauer, B. (2018). Image and reputation of family firms: A systematic literature review of the state of research. Review of Managerial Science, 12(1), 335-377. https://doi.org/10.1007/s11846-016-0 216-x 
Shamil, M. M., Shaikh, J. M., Ho, P. L., \& Krishnan, A. (2014). The influence of board characteristics on sustainability reporting: Empirical evidence from Sri Lankan firms. Asian Review of Accounting, 22(2), 78-97. https://doi.or g/10.1108/ARA-09-2013-0060

Shaukat, A., Qiu, Y., \& Trojanowski, G. (2016). Board attributes, corporate social responsibility strategy, and corporate environmental and social performance. Journal of Business Ethics, 135(3), 569-585. https://doi.org/10.1007/s 10551-014-2460-9

Superintendencia Financiera de Colombia (2007). Código País. Sección 2. Junta Directiva subsección 2.1 Tamaño, conformación y funcionamiento. Recuperado de https://www.superfinanciera.gov.co/SFCant/Codigopais/jun tadirectiva.htm

Zahra, S. A., \& Pearce, J. A. (1989). Boards of directors and corporate financial performance: A review and integrative model. Journal of Management, 15(2), 291-334. https://doi.org/10.1177/014920638901500208

\section{Notas}

* Artículo de investigación.

1 http://database.globalreporting.org/search. Consulta el 20 de abril de 2017.

2 Encuesta recuperada de https://www.superfinanciera.gov.co/publicacion/10782

3 De acuerdo con lo dispuesto por el artículo 11.2.1.6.2 del Decreto 2555 de 2010 "son emisores de valores las entidades que tengan valores inscritos en el Registro Nacional de Valores y Emisores en Colombia”.

$4 \quad$ Statistical Tool for Analyzing Trends in Social and Environmental Performance

5 http://www.merco.info/

6 Es importante mencionar que a la fecha de la consulta en julio de 2016, se podía acceder al portal empresarial de la Supersociedades en el siguiente link http://portalempresarial.supersociedades.gov.co/Paginas/ConsultaSociedad.aspx sin embargo, en la actualidad el sistema cambió y ahora se accede en http://sie.supersociedades.gov.co/(S(xvmvfndsuk ocy1rwur5yypmo))/Pages/Default.aspx/empresa

7 Allí se establecen, entre otras, las siguientes condiciones (de independiente): que no sea empleado o directivo de la compañía, accionista, socio o empleado de asociaciones o sociedades que presten servicios a la compañía o empleado o directivo de una fundación que reciba aportes de la compañía http://www.alcaldiabogota.gov.co/sisjur/normas/Norm a1.jsp?i=22412

\section{Licencia Creative Commons CC BY 4.0}

Para citar este artículo: Wilches S., A., Orozco, L. A., \& Beltrán-Torres, C. Y. (2020). Estructura de las juntas directivas y divulgación de la responsabilidad social corporativa: el caso de las empresas más reputadas en Colombia. Cuadernos de Administración, 33. https://doi.org/10.11144/Javeriana.cao33.ejdd 